1 Universidade Federal do Rio Grande do Norte (UFRN), Escola Multicampi de Ciências Médicas, Programa de PósGraduação em Ensino na Saúde - Caicó (RN), Brasil. vianacostam@yahoo.com.br

2 Universidade Federal do Rio Grande do Norte (UFRN), Escola Multicampi de Ciências Médicas, Programa de PósGraduação em Educação, Trabalho e Inovação em Medicina - Caicó (RN) Brasil.

3 Universidade Federal do Rio Grande do Norte (UFRN), Centro de Ciências da Saúde, Programa de PósGraduação em Ensino na Saúde - Natal (RN), Brasil.

\section{Aspectos institucionais para a adoção da Educação Interprofissional na formação em enfermagem e medicina}

\author{
Institutional aspects for the adoption of Interprofessional Education \\ in nursing and medical training
}

Marcelo Viana da Costa', George Dantas Azevedo², Maria José Pereira Vilar ${ }^{\mathbf{3}}$

DOI: 10.1590/0103-11042019S105

RESUMO Os marcos teórico-conceituais e metodológicos da Educação Interprofissional (EIP) em saúde vêm sendo amplamente reconhecidos em todo o mundo como úteis para o desenvolvimento de competências colaborativas para o efetivo trabalho em equipe. $\mathrm{O}$ artigo teve como objetivo explorar as percepções de estudantes de enfermagem e medicina sobre os fatores institucionais que interferem na adoção de iniciativas de EIP em seus contextos de formação. Trata-se de um estudo de caso, que adotou a abordagem qualitativa e a perspectiva exploratória. Os participantes da pesquisa foram estudantes de enfermagem e medicina de duas universidades públicas de um estado do Nordeste - uma estadual e outra federal. O grupo focal foi escolhido para coleta de dados, e foi utilizada a técnica de análise de conteúdo categorial, observando as fases de pré-análise, exploração do material e tratamento dos resultados. Três categorias temáticas foram construídas a posteriori: a importância do trabalho em equipe, contextos institucionais para adoção da EIP e desafios para a adoção da EIP. Embora as realidades pesquisadas apresentem avanços nas mudanças curriculares, como a aproximação do ensino com a realidade dos serviços, a adoção de métodos mais ativos para a formação de sujeitos críticos e reflexivos, ainda são notórias as lacunas no desenvolvimento de competências colaborativas.

PALAVRAS-CHAVE Educação superior. Instituições acadêmicas. Relações interprofissionais.

\begin{abstract}
The theoretical-conceptual and methodological frameworks of Interprofessional Education in Health (IPE) have been widely recognized throughout the world as useful for the development of collaborative competences for effective teamwork. This article aims to explore the perceptions of nursing and medical students regarding the institutional factors that interfere in the adoption of IPE initiatives in their training contexts. It is a case study, which adopted the qualitative approach and the exploratory perspective. The research participants were nursing and medical students from two public universities from a Brazilian Northeastern state - one state and one federal. The focus group was chosen for data collection, and the categorical content analysis technique was used, observing the phases of pre-analysis, material exploration and treatment of results. Three thematic categories were built a posteriori: the importance of teamwork, the institutional contexts for the adoption of the IPE and challenges for the adoption of the IPE. Although the
\end{abstract}


realities researched present advances in curricular changes, such as the approximation of teaching with the reality of services, the adoption of more active methods for the formation of critical and reflexive subjects, the gaps in the development of collaborative competences are still notorious.

KEYWORDS Education. Higher, schools. Interprofessional relations.

\section{Introdução}

A Educação Interprofissional (EIP) vem sendo discutida, ao longo dos últimos 30 anos, como forma de incentivar novas relações entre os profissionais de saúde, por meio da colaboração, e, consequentemente, melhorar a qualidade dos serviços de saúde. Nesse período, muitos são os esforços para consolidar o debate, superando os equívocos teóricos, identificar evidências de sua efetividade na mudança de atitudes e habilidades dos profissionais de saúde, transformar a realidade dos sistemas de saúde, construir um marco teórico e discutir metodologias e estratégias que subsidiem a lógica da EIP'.

Estudiosos e pesquisadores, a partir de importantes acúmulos históricos, empenharam-se na construção de uma definição capaz de atender às demandas emergentes no campo da formação dos profissionais da saúde, na necessidade de superar o grande distanciamento entre os profissionais da saúde e no compromisso de melhorar a qualidade da atenção e a qualidade de vida e saúde das pessoas ${ }^{\mathbf{2}, 3}$.

O Center For The Advancement of Interprofessional Education (Caipe) ${ }^{\mathbf{4}(2)}$, importante instituição que incentiva e apoia a EIP no Reino Unido, defende que a

Educação Interprofissional ocorre quando duas ou mais profissões aprendem com, para e sobre a outra, para melhorar a colaboração e a qualidade dos cuidados.

Estudo de revisão sistemática, por sua vez, buscando dar destaque aos aspectos centrais da interprofissionalidade, defende que a EIP

[...] ocorre quando os membros de mais de uma profissão da saúde e/ou assistência social aprendem em conjunto, de forma interativa, com o propósito explícito de melhorar a colaboração interprofissional e/ou a saúde/ bem-estar dos pacientes/clientes ${ }^{5(5)}$.

Aspecto central dessa definição é a intencionalidade para o desenvolvimento de competências colaborativas. Oportunidades de aprendizagem entre estudantes ou profissionais de diferentes categorias devem ser potencializadas por estratégias intencionalmente planejadas para que a colaboração substitua a tradicional competição existente na formação e no trabalho em saúde.

Nas definições sobre EIP, é possível perceber a evolução do entendimento de que a formação do profissional acontece em diversos cenários, tanto na realidade da produção dos serviços de saúde quanto nos espaços formais e informais de formação da força de trabalho em saúde, assim como da importância desse processo ser compartilhado pelos atores envolvidos, estudantes e/ou profissionais ${ }^{3}$.

Outro aspecto que chama atenção é que o amadurecimento do debate permitiu definir a EIP como pressuposto para o trabalho colaborativo, colocando na centralidade do processo as necessidades sociais e de saúde, dos usuários; bem como superar o atual modelo de formação em silos profissionais, em que há pouca ou nenhuma oportunidade de aprendizagem 
compartilhada, e que acaba por determinar práticas também separadas e isoladas ${ }^{6}$.

Discutir educação e o trabalho interprofissional retoma a reflexão sobre a centralidade dos usuários na elaboração e execução das ações em saúde, mostrando-se coerente com o propósito de fortalecer os sistemas de saúde por meio de respostas mais efetivas aos problemas de saúde. Reaviva o debate em torno do diálogo necessário entre os diversos campos de conhecimento e de práticas para o enfrentamento de problemas e necessidades de saúde que se tornam complexos em uma dinâmica social que também precisa de múltiplos olhares e ações.

Tomando por base a relevância da EIP para a formação de profissionais de saúde mais aptos à colaboração e ao efetivo trabalho em equipe, a elaboração deste estudo foi orientada pela seguinte questão norteadora: como estudantes de enfermagem e medicina percebem os aspectos institucionais para a adoção da EIP em seus contextos de formação?

Dessa forma, o artigo tem como objetivo explorar as percepções de estudantes de enfermagem e medicina sobre os fatores institucionais que interferem na adoção de iniciativas de EIP em seus contextos de formação.

\section{Material e métodos}

Fundamentado na natureza do problema pesquisado e nos objetivos estabelecidos, trata-se de um estudo de caso. Embora o estudo tenha acontecido com participantes de diferentes instituições de ensino, adotam-se como caso para estudo os aspectos institucionais que influenciam na adoção da interprofissionalidade na formação em enfermagem e medicina. Dessa forma, considera-se o contexto institucional como "um sistema delimitado e contemporâneo da vida real"7(32).

Considerando a classificação em relação à abordagem e ao alcance dos objetivos, trata-se de uma pesquisa tanto qualitativa quanto exploratória. A abordagem qualitativa se mostrou adequada na medida em que permite compreender o fenômeno para além das suas características mensuráveis e a capacidade de compreender, discutir e explorar sensações e experiências existentes nas relações sociais ${ }^{8}$. A perspectiva exploratória justifica-se pela intenção de explorar aspectos de um determinado fenômeno, principalmente quando ainda não se conhece bem os aspectos que o constituem ${ }^{9}$.

O estudo foi desenvolvido em duas universidades de um estado do Nordeste - uma estadual e outra federal. Entre as duas, há importantes diferenças na estrutura, organização, interiorização e em relação aos processos de pesquisa, ensino e extensão. A escolha foi motivada pelo fato de que as duas universidades têm tradição na participação em políticas de reorientação da formação profissional em saúde.

Os participantes da pesquisa foram estudantes de enfermagem e medicina de duas universidades públicas localizadas no Nordeste brasileiro. Para assegurar a confidencialidade dos participantes, foram utilizados códigos compostos pelas letras EE - para Estudantes de Enfermagem - e pelas letras EM - para Estudantes de Medicina - seguido de um numeral.

O grupo focal foi escolhido para coleta de dados por levantar questões relacionadas com a disponibilidade para a aprendizagem compartilhada, estereótipos profissionais, limitações ou dificuldades para o aprendizado interprofissional. Os grupos focais foram homogêneos, e esse formato permitiu aos participantes a oportunidade de fornecer informações mais concretas em seus pontos de vista sobre estudantes de outras áreas profissionais $^{\mathbf{1 0}}$.

A discussão no grupo focal foi conduzida pelo moderador, coordenador da pesquisa, e por um observador, responsável pelo registro de atitudes e gestos que possam complementar a observação e análise de conteúdo ${ }^{11-13}$. Com o intuito de problematizar a realidade dos serviços de saúde e levantar questões referentes aos aspectos institucionais que interferem na adoção da EIP na formação em saúde para o desenvolvimento de competências para o trabalho colaborativo, a discussão nos grupos 
foi orientada por algumas questões iniciais: qual a importância do trabalho em equipe para o trabalho em saúde? Que aspectos da realidade da formação se aproximam da EIP? Quais aspectos institucionais facilitam ou dificultam a EIP?

Em relação ao tamanho e à duração do grupo, a média foi de oito participantes, e a duração variou de 50 a 80 minutos. A escolha de um pequeno grupo justifica-se pela possibilidade de viabilizar maior interação entre os participantes, facilitar a comunicação e discussão das questões levantadas.

Foram realizados quatro grupos focais na universidade estadual, sendo dois grupos em cada curso, com um total de 33 participantes. Na universidade federal, foram realizados dois grupos focais em enfermagem (primeira e segunda metade) e um grupo focal com estudantes da primeira metade do curso de medicina, totalizando três grupos focais, com 25 participantes nesses grupos. Os grupos focais foram gravados e, em seguida, transcritos, compondo o corpus de análise da pesquisa.

A realização da técnica de análise respeitou as fases de pré-análise, exploração do material e tratamento dos resultados. Na pré-análise, foi realizada a leitura flutuante que permitiu o contato inicial com os documentos, o conteúdo obtido, a partir das falas dos grupos focais e entrevistas. As transcrições de cada grupo focal e entrevista foram lidas para obter uma imersão no discurso, tendo uma primeira aproximação das mensagens, argumentações e justificativas que têm contribuído para uma maior clareza das hipóteses, permitindo maior clareza das principais mensagens $\mathbf{1}^{\mathbf{1 2}}$.

Em seguida, procedeu-se à 'constituição do corpus'. Nessa etapa, foi observada a regra de exaustividade, e foram organizados os dados para responder a padrões de validade, contemplado todos os aspectos levantados na discussão, sem omissão de nenhum aspecto; representatividade, que assegurou que os dados (mensagens, falas, expressões) fossem representativos da totalidade; homogeneidade, que obedeceu a critérios precisos de escolha, assegurando que os dados se referissem ao mesmo tema, coletados por meio de técnicas iguais em indivíduos semelhantes; e pertinência, que possibilitou reconhecer que o corpus foi adequado aos objetivos do trabalho ${ }^{\mathbf{1 4}}$.

$\mathrm{Na}$ terceira etapa, foi realizada a 'formulação de hipóteses e objetivos' que assegurou realizar algumas afirmações provisórias, a partir das fases anteriores. A formulação dos objetivos foi feita a partir da seleção das unidades de análise, por meio do processo de categorização, a posteriori ou empírico, em que as categorias que emergiram foram descritas e discutidas a partir do referencial teórico existente ${ }^{12,14}$.

Já a última etapa foi a preparação do material, antes da análise propriamente dita. Nesse momento, foi feita a numeração dos elementos do corpus, assim como a identificação das unidades de análise, que, para este estudo, foi destacada com cores diferenciadas.

As etapas de exploração do material, tratamento e interpretação dos resultados foram facilitadas pelas etapas, e suas fases, que as precederam. As unidades de análises foram codificadas, decompostas e enumeradas, facilitando a interpretação desses resultados a partir dos objetivos propostos pela pesquisa.

Este estudo foi registrado e aprovado pelo Comitê de Ética do Hospital Universitário Onofre Lopes da Universidade Federal do Rio Grande do Norte, por meio do parecer de $n^{0} 16.652$, que observou os objetivos da pesquisa, riscos e benefícios a que os participantes foram submetidos.

\section{Resultados}

Os dados dos grupos focais trouxeram características importantes sobre a realidade da formação em saúde para a adoção da EIP. Assim, o corpus da pesquisa permitiu agrupar os resultados em categorias temáticas de análise, subcategorias e unidades de análise, conforme sumarizado na tabela 1 . 
Tabela 1. Categorias de análise obtidas pelos grupos focais

\begin{tabular}{|c|c|c|}
\hline Categorias & Subcategorias & $\begin{array}{r}\text { Unidades de } \\
\text { análise }\end{array}$ \\
\hline \multirow[t]{4}{*}{ Importância do Trabalho em Equipe } & Atenção Integral & 14 \\
\hline & Integração de Conhecimentos/Profissionais & 15 \\
\hline & $\begin{array}{l}\text { Dificuldade de Interação na Lógica do Modelo } \\
\text { Assistencial }\end{array}$ & 8 \\
\hline & Intensa Hierarquia & 5 \\
\hline \multirow[t]{6}{*}{ Os contextos institucionais para a EIP } & Atividades de Ensino e Extensão & 11 \\
\hline & $\begin{array}{l}\text { Atividades de Ensino/Aprendizagem compartilha- } \\
\text { dos sem sistematização }\end{array}$ & 16 \\
\hline & $\begin{array}{l}\text { Atividades Interdisciplinares (áreas de conhecimen- } \\
\text { to de um único curso) }\end{array}$ & 8 \\
\hline & Corpo Docente reproduz a separação das áreas & 21 \\
\hline & Iniciativas Individuais (professores ou estudantes) & 08 \\
\hline & Confusão Conceitual & 08 \\
\hline \multirow[t]{4}{*}{ Desafios para a adoção da EIP } & Espaço Físico/Estrutura Física das Universidades & 16 \\
\hline & $\begin{array}{l}\text { Muita Ênfase na Formação Específica/Modelo } \\
\text { Tradicional de Formação }\end{array}$ & 19 \\
\hline & Hierarquia/Cultura dos Papéis Profissionais & 09 \\
\hline & Estereótipo/Pouco conhecimentos das outras áreas & 08 \\
\hline
\end{tabular}

Fonte: Elaboração própria.

\section{Importância do trabalho em equipe}

Nos grupos focais, os estudantes demonstraram boa compreensão da relevância do trabalho em equipe, reiterando a interação entre os profissionais como um elemento importante para melhorar a qualidade do atendimento e obtenção de melhores resultados para os problemas e necessidades de saúde. A necessidade de uma atenção integral apareceu de forma muito frequente nas falas e discussões.

Nós já vimos na teoria a importância do trabalho em equipe para prestar um serviço mais qualitativo, para melhorar a resolutividade para o usuário, e com a prática, vimos a importância da integração dos profissionais nas equipes. (EM1).

A assistência ao paciente tem que ser um integral. Não podemos - enfermagem, medicina, fisioterapia - nenhuma dessas profissões fornecem um atendimento integral ao paciente que responda às necessidades, sendo independentes uns dos outros. (EE1).

Os estudantes destacaram com frequência a importância do trabalho em equipe como oportunidade de troca de conhecimentos e construção de novos conhecimentos, demonstrando a necessidade de diálogo entre os sujeitos e superando o entendimento da equipe que apenas ocupa o mesmo espaço, sem a comunicação necessária para a efetivação do trabalho em equipe. Trouxeram também a importância da centralidade do usuário/ paciente e da necessidade de um objetivo em comum, capaz de viabilizar a interação entre os diferentes profissionais.

Eu acho que quando todos os profissionais que trabalham em conjunto e priorização por um objetivo comum é um trabalho de equipe. $E$ isso é necessário. (EE2). 
Eu acho que nenhum trabalho que você vai fazer, envolvendo qualquer área, você não consegue fazer sozinho, é preciso que todos colaborem um pouco, que todos se comuniquem. Eu acho que a questão fundamental também do trabalho em equipe é a comunicação e uma boa convivência. (EM6).

Embora tenha sido possível identificar a maturidade dos estudantes no debate, o diálogo como um elemento importante para permitir o trabalho colaborativo apareceu de forma mais enfática em apenas um grupo. A capacidade de diálogo surge na discussão enquanto competência que varia de acordo com a abertura individual e independentemente da categoria profissional. Os participantes relataram que há deficiências nas relações entre os diferentes profissionais, dificultando o trabalho em equipe na perspectiva da colaboração.

A classe médica de uma forma geral [... é colocada num pedestal de um lado e os demais profissionais do outro lado. Então tem um embate muito grande entre os profissionais médicos e as demais categorias. (EM8).

Na equipe, cada profissional tem o seu ponto de vista. O profissional vê o problema do seu ponto de vista e cada membro da equipe pode acumular o que ele pensa com o que os outros pensam, e eles vão chegar a um bem comum. Porque às vezes nós somos muito focados no que fazemos e deixamos de considerar as contribuições dos outros dentro da equipe. (EE6).

\section{Contextos institucionais para adoção da EIP}

A maioria dos participantes afirmou nunca ter ouvido falar sobre a EIP. Quando instigados a falar sobre a aproximação com o debate da EIP, os estudantes, apesar de mostrarem pouco conhecimento da discussão, tentaram explicá-la a partir do prefixo 'inter'. Na discussão, fizeram referência à interdisciplinaridade e ao trabalho multiprofissional. Um pequeno grupo de estudantes de medicina afirmou conhecer o tema, no entanto, durante o debate, demonstraram confusão conceitual, apontando o entendimento da multiprofissionalidade, multidisciplinaridade e interdisciplinaridade como termos sinônimos.

Educação Interprofissional?! Este tema, eu nunca ouvi. (EE5).

O que vem à mente é a interação entre as profissões. (EE3).

Trabalho interdisciplinar a gente já escutou [...], mas realmente sermos educados todos juntos de forma interprofissional não! (EM4).

A pouca aproximação com a discussão não é suficiente para assegurar que na realidade pesquisada não seja adotada nenhuma iniciativa na direção do desenvolvimento das competências colaborativas. Partindo desse pressuposto, os participantes foram estimulados a falar sobre as estratégias/ações que tentaram trazer estudantes de cursos diferentes no processo de formação, como forma de qualificar os estudantes para o trabalho em equipe.

Atividades de ensino e projetos de pesquisa e de extensão foram citados como experiências que aproximam estudantes de diversos cursos. Nas falas, foi possível identificar importante variedade de ações e de objetivos. Em alguns relatos, percebe-se a intencionalidade da interação, da troca de experiências e aproximação entre os sujeitos.

Nós temos duas disciplinas que são feitas para essa integração. São estudantes de fisioterapia, nutrição, farmácia, medicina e enfermagem e serviço social às vezes. Estes estudantes se encontram em um estabelecimento de saúde e pensam juntos sobre como agir nessa comunidade. (EE1).

Um dos lugares que a gente mais aprendeu [...] foi participar do Programa de Educação pelo Trabalho em Saúde (PET), que tinha alunas de 
enfermagem também. A gente teve que se unir, teve que colaborar um com o outro e nisso algumas visões foram quebradas, tanto deles quanto da gente. (EM6).

As iniciativas sistematizadas de aprendizagem compartilhada mencionadas pelos participantes ainda estão fundamentadas na lógica multidisciplinar e multiprofissional, refletindo as grandes dificuldades para realizar a interação entre as diferentes profissões. A inexistência de ações intencionais e sistematizadas podem ser entendidas como barreiras para a incorporação dos marcos teórico-conceituais e metodológicos da EIP.

Eu estava pensando... Eu não vejo nenhum momento de nossa formação contribuir para este trabalho em equipe... (EE11).

Eu não vejo uma preocupação, desde o início da faculdade, em trabalhar em equipe. Na verdade [...] você passa a considerar o ser humano de uma forma geral, de uma forma holística, mas em nenhum momento passaram pra gente como trabalhar em equipe. (EM7).

E o que eu acho também, em relação ao currículo da gente, que falta muito. A gente é muito ignorante sobre o que o outro faz, sobre o que o fisioterapeuta faz, quais são as atribuições do fisioterapeuta, do enfermeiro, do fonoaudiólogo. (EM8).

Os estudantes expõem dois aspectos da realidade: dificuldades na implementação de iniciativas sistematizadas e ausência completa de ações que objetivem a interação de estudantes de diferentes cursos para desenvolver competências colaborativas. Os participantes relatam que o encontro com outros estudantes, muitas vezes, acontece por acaso e sem planejamento, dificultando ainda mais o fortalecimento das relações interpessoais e interprofissionais.

No leito quando chegamos, o paciente estava com os estudantes de medicina, ficamos esperando eles terminarem e então começamos nosso atendimento. (EE11).

Na semana passada nós estávamos fazendo uma intervenção com uma criança no leito e chegaram estudantes de fisioterapia. Eles ficaram esperando terminarmos a intervenção para entrar. Os conhecimentos de enfermagem estavam conosco e o conhecimento de fisioterapia estava depois da porta e não conseguimos articular. (EE14).

Durante a faculdade de medicina não é explorado, eu sinto essa necessidade gritante no nosso curso, trabalhar mesmo de uma forma mais integrada. (EM7).

Também apareceu nas falas que nos momentos em que os estudantes se encontram por acaso ou coincidência, por dividirem os mesmos espaços de aulas práticas, a interação encontra fortes barreiras estruturais e culturais. Mencionaram também que, quando acontece, é por iniciativa individual dos estudantes. Destacam o interesse que essa interação acontecesse como um processo natural na efetivação do trabalho em saúde.

Realmente, se não fosse a minha iniciativa, não haveria articulação. Não teria essa interação mútua. (EE2).

A interação que existiu foi iniciativa dos estudantes. (EM7)

Essa articulação é muito mais favorecida em estágio supervisionado. Mas depende de cada aluno. (EE12).

A realidade apresentada pelos participantes dos grupos focais é complexa e chama a atenção para a atuação docente no processo de formação. As relações entre os professores de diferentes departamentos apareceram como um aspecto que caminha na contramão da formação de competências para o trabalho colaborativo.

Eu acho que o grande problema é que não 
estamos sendo formados para trabalhar em equipe. O que custa o professor de uma disciplina sentar com o professor de fisioterapia e tentar articular as agendas de ações para ser feito juntos? Vemos isso dentro de faculdade. Muitas vezes fazemos um trabalho duas vezes, porque a equipe diz que é interdisciplinar, mas não consegue se sentar para discutir. Vemos as diferenças dentro da equipe da mesma disciplina. Nós ainda não estamos sendo formados por uma equipe. Então, o profissional não sai preparado para trabalhar em equipe. Apesar do discurso de equipe. (EE10).

Os professores são muito desarticulados. Até mesmo os que estão em um mesmo departamento. A gente percebe essa desarticulação nas provas. Em disciplinas compartilhadas a gente sabe quem elaborou cada questão. (EM15).

\section{Desafios para a adoção da EIP}

Os relatos dos estudantes desenham uma realidade de importantes pontos positivos para a adoção da EIP, como abordagem para a reorientação da formação em saúde. No entanto, quando narram as experiências vivenciadas, expõem importantes desafios para que as realidades avancem na perspectiva da formação de profissionais mais aptos ao trabalho colaborativo na produção dos serviços de saúde.

As iniciativas, apesar de apresentarem problemas com a execução, constituem-se como espaços potentes para a construção de estratégias baseadas na EIP. No entanto, muitos desafios foram relatados pelos estudantes. Diante dos aspectos que emergiram na técnica de coleta dos dados, as dificuldades mencionadas são de diversas esferas e evidenciam a necessidade de pensar o papel da universidade.

Um importante desafio trazido por estudantes de medicina foi a desarticulação entre teoria e prática. De acordo com os participantes, o debate que acontece em sala de aula aborda alguns pontos por ocasião de aulas práticas, mas a realidade vivenciada pelos estudantes nos serviços de saúde é diferente, transformando o debate do trabalho em equipe em quimera.

O sistema [de saúde] não pensa de forma multiprofissional. Então, nós nunca seremos multiprofissionais. A mentalidade de sistema é este e não temos como ir contra. Não adianta gastar trinta aulas falando de uma equipe multiprofissional, se quando vamos lá, você não vê multiprofissional. Na sala de aula vemos de uma forma superficial. Aprendemos mesmo é na realidade. (EM1).

Temos no início, as partes teóricas. Professores dizem que o cuidado deve ser centrado no paciente. Tem a iniciativa. É o começo. Mas quando vamos para os serviços, percebemos que é diferente. Ficamos entre fazer o que aprendemos ou fazer como conseguimos ver na realidade dos serviços de saúde. Nós achamos que vamos colocar tudo que aprendemos em prática, mas quando chegamos lá, temos que nos moldar um pouco. (EE4).

A estrutura física, frequentemente, apareceu como um dos elementos que prejudica a interação entre estudantes de diferentes cursos com o objetivo de aprendizagem compartilhada. Ambas as realidades apresentam limitações importantes da estrutura física que acentuam os problemas já relatados. As faculdades têm edifícios próprios e em estruturas separadas, muitas vezes distantes, prejudicando o diálogo entre estudantes e professores.

O departamento de medicina está muito longe. A farmácia também. Eu acho que isso faz com que seja mais difícil. Como posso falar com alguém, se eu nem mesmo o conheço? (EE4).

A gente tem o nosso prédio que é nomeado faculdade de ciências da saúde e só funciona o curso de medicina. (EM5).

Outro grande desafio relatado nos grupos é a identidade profissional e os papéis estabelecidos historicamente. Assim, o debate sobre a profissionalização ganha espaço como uma 
maneira de entender como as profissões adquirem identidades que acabam definindo, também, as possibilidades de relações com outras profissões.

Estudantes do Curso de Medicina reconhecem que há uma barreira cultural para o diálogo com outros grupos profissionais. Essa barreira é legitimada tanto pela categoria como por usuários que atribuem à ação uniprofissional, segundo relatos, maior confiança no atendimento. Estabelecer diálogos com outros profissionais pode expressar insegurança profissional.

Estudantes de enfermagem também referem a existência de resistências entre os estudantes de medicina e enfermagem. Eles também afirmam que a naturalização da hierarquia e de relações verticais existe na própria equipe de enfermagem.

Nós não vamos negar que existe entre os médicos um certo preconceito de dizer que precisa de uma segunda opinião de outro profissional que não seja médico. Isso é fato. (EM2).

Existem certas formações que isola o profissional, como se apenas o trabalho de uma categoria profissional fosse melhor. Nós encontramos resistências. Isso é uma visão limitada que não permite a articulação do nosso trabalho. (EE3).

\section{Discussão}

Embora se reconheça a necessidade de estabelecer limites às profissões, o cenário atual, de grandes e importantes transformações, demanda um novo profissionalismo em que os profissionais estejam aptos ao trabalho em equipe e comprometidos com o fortalecimento dos sistemas de saúde e capazes de estabelecer novas relações entre os usuários dos serviços de saúde ${ }^{15}$.

O entendimento dos participantes demonstra a aproximação com a literatura em torno dos benefícios e necessidade de trabalho em equipe, embora haja uma variedade de definições para esse tipo de trabalho. Em todas as definições, entretanto, reiteram a interação entre os profissionais como prerrogativa para atender às complexas e dinâmicas necessidades de saúde, em tempos atuais ${ }^{16}$.

A essência desse trabalho, em que a colaboração assume papel central, coloca o usuário dos serviços, e suas necessidades, como fundamental no processo de produção dos serviços de saúde. A complexidade e a natureza dos problemas e necessidades dos usuários sugerem a necessidade de complementariedade, a partir da comunicação, para compartilhar experiências e conhecimentos, capazes de orientar a tomada de decisões, para o enfrentamento dos problemas apresentados ${ }^{17}$. Pesquisas recentes indicam que o trabalho em equipe na perspectiva de colaboração favorece a segurança do paciente, a partir da centralidade de suas necessidades ${ }^{5,18}$.

Tomando por base essa problemática, estudos e experiências vivenciadas em diversos países defendem a EIP como importante instrumento para a mudança do foco das práticas profissionais, superando modelos centrados em suas especificidades, como forma de possibilitar processos de aprendizagem compartilhados, capazes de estimular a melhoria das competências colaborativas ${ }^{19}$.

A EIP em saúde almeja criar, no processo de aprendizagem, condições para melhorar as relações entre membros de diferentes profissões, superando a dificuldade histórica de comunicação, possibilitando a formação de sujeitos aptos a trabalharem de forma colaborativa, na atenção à saúde das pessoas. A EIP se constitui, então, como proposta de superação do modelo de formação, pautado da perspectiva multiprofissional ou uniprofissional ${ }^{5}$, que tem apresentado muitas limitações na formação de profissionais capazes de atender às demandas que emergem no contexto atual.

Dentre as barreiras ou desafios, está a necessidade de pensar a logística e o reconhecimento da importância de um planejamento rigoroso e a necessidade de recursos para a 
obtenção dos resultados esperados; os desenhos curriculares se apresentam como outra barreira, na medida em que estão organizados, a partir das necessidades de formação específica de cada profissão, exigindo maior esforço para a negociação e flexibilidade desses desenhos; a cultura atual que reforça os limites profissionais e o diálogo e interação entre as diferentes categorias profissionais e as relações de aprendizagem muito focadas em perspectivas tradicionais que pouco contribuem para a efetivação da colaboração e interação ${ }^{\mathbf{2 0}}$.

Ponto fundamental para discutir o contexto das realidades pesquisadas para a adoção da EIP é o apoio institucional às iniciativas que se estimulam mudanças nos níveis macro, meso e micro da realidade ${ }^{21}$. Aspectos dos domínios micro e médio podem comprometer seriamente as iniciativas implementadas, o que aponta para a compreensão de que as mudanças devem acontecer em um processo articulado e planejado, em exaustão, para garantir impacto nos problemas da formação profissional em saúde 20,22 .

Da mesma forma, apesar da existência de políticas (nível macro) que incentivem a reorientação da formação profissional em saúde, há importantes lacunas na adoção de estratégias (domínio médio) capazes de superar as relações estabelecidas no trabalho em saúde, como relatado pelos participantes da pesquisa, que podem minimizar as barreiras impostas pelas estruturas físicas nos contextos pesquisados.

Percebe-se, então, a importância dos aspectos do domínio meso ${ }^{21}$ para a efetivação das mudanças. Estudantes sentem a necessidade de maiores estímulos à construção de novas competências, a fim de avançar na formação interprofissional. No entanto, não é tarefa simples elaborar atividades interprofissionais porque envolve grandes esforços para superar não apenas a barreira física, mas também pensar em estratégias possíveis, diante das especificidades de cada curso: número de estudantes, diferentes desenhos curriculares, cronograma de atividades muito distintas e que são construídos no interior das faculdades ou departamentos ${ }^{21}$, sem a necessária articulação ou diálogo, o que pode ser visto nas duas universidades estudadas.

A realidade estudada indica a existência de momentos, planejados e não planejados, nos quais estudantes de diferentes cursos se unem, mas que têm se aproximado muito mais da perspectiva multiprofissional. As iniciativas existentes são poucas, ou inexistentes, e sofrem com a falta de planejamento, que culmina na reprodução do modelo tradicional de formação, formando indivíduos pouco comprometidos com a transformação do atual cenário da educação e da atenção à saúde.

As atuais políticas de reforma do processo de formação dos profissionais de saúde têm possibilitado a inserção dos estudantes de todas as áreas nos serviços de saúde, considerando espaço adequado para a formação de profissionais mais críticos e reflexivos. No entanto, a realidade marcada pela forte divisão do trabalho não estimula, na academia, a construção de competências para o trabalho colaborativo. O professor pode aproveitar os espaços da realidade concreta para criar um espaço adequado para a colaboração entre os estudantes. Para tanto, o professor precisa incorporar a importância da EIP para a obtenção das transformações necessárias ${ }^{23}$, nas realidades estudadas.

Já em relação à dimensão micro, merece discussão o processo de construção de identidades profissionais que acaba por delinear papéis, valores e atitudes que vão muito além da educação e processos de formação ${ }^{\mathbf{2 4}}$. A sociedade incorpora essas identidades, fortalecendo ainda mais as barreiras ao diálogo. Este processo contribui para que o diálogo e a interação se tornem cada vez mais difíceis. As identidades profissionais, historicamente construídas, edificam barreiras fortes para a interação interprofissional ${ }^{25,26}$. É interessante notar que as falas dos estudantes mantêm aderência com a literatura. 
A literatura e a realidade pesquisada destacam questões que merecem atenção no debate sobre a EIP: relações de poder, que ganham força pela hierarquia profissional, e relações de gênero, que estiveram presentes nos debates sobre as interações entre médicos e enfermeiros ${ }^{27}$. O processo de formação, que se configura como instrumento útil para alcançar as transformações necessárias, deve rever a maneira como as relações hierárquicas vêm sendo abordadas e de que forma as especificidades das categorias profissionais justificam esse formato de relações ${ }^{\mathbf{2 8}}$.

Estudos mostram que estudantes da área da saúde que participam de atividades, na perspectiva da EIP, desenvolvem melhor habilidades de comunicação e trabalho em equipe, dispõem de mais clareza sobre os papéis dos diversos profissionais que compõem a equipe e se mostram mais aptos no gerenciamento de conflitos que possam surgir na dinâmica do trabalho em saúde ${ }^{29}$. No que diz respeito às relações de poder, é possível dizer que a dificuldade de diálogo e trabalho colaborativo foi tomando forma ao longo da história, legitimada pela ideia de superioridade de uma profissão sobre a outra ${ }^{30}$.

Pontos como respeito, conhecimento dos papéis dos colegas de trabalho e hierarquia são vistos, ainda, como problemáticos, reforçando ainda mais a necessidade de pensar em estratégias, no processo de formação, que estimulem e incentivem novos hábitos e atitudes para o efetivo trabalho em equipe.

\section{Considerações finais}

Embora a realidade apresente importantes avanços nas mudanças curriculares, como a aproximação do ensino com a realidade dos serviços e a adoção de métodos mais ativos para a formação de sujeitos críticos e reflexivos, ainda são notórias as lacunas no desenvolvimento de competências colaborativas. A EIP se apresenta como abordagem útil, e necessária, para as realidades pesquisadas, com vistas a superar as fortes barreiras estabelecidas para a melhoria das relações e interações interprofissionais.

A inserção e o fortalecimento do tema nos contextos das instituições de ensino superior pesquisadas não implicam apenas a adoção do termo, de forma indiscriminada, nos documentos oficiais. Não pode se configurar como modismo, ou como mera condicionalidade em processos de avaliação. Exige um comprometimento desde os formuladores das atuais políticas de saúde até a atuação de estudantes e professores, nos espaços de ensino e aprendizagem.

Não se trata de uma tarefa simples, pelas fortes barreiras culturais incorporadas no âmbito na universidade, das categorias profissionais e da própria sociedade. A EIP evidencia a necessidade de discutir a forte ideia de hierarquia, a histórica soberania de áreas de conhecimentos ou de categorias profissionais e a urgência em (re)situar os sujeitos, atribuindo-lhes centralidade, o que não é estranho para o sistema de saúde brasileiro, pensado a partir da integralidade, universalidade e equidade. Aspectos esses muito presentes na realidade de formação demonstrada nesta pesquisa a partir da percepção dos estudantes participantes.

As iniciativas existentes nas duas universidades, induzidas pelas atuais políticas ou pelo acúmulo histórico em torno do processo de reorientação da formação profissional em saúde, devem ser fortalecidas e aperfeiçoadas para assegurarem uma formação profissional sob novas bases; e as instituições devem se constituir enquanto espaço legítimo de construção coletiva de novos projetos de universidade, de sociedade e de saúde. Dessa forma, e, por tudo que foi debatido aqui, é que se acredita no potencial da EIP e no trabalho colaborativo como ferramentas necessárias para esses projetos.

É mister inserir o debate sobre EIP nas atuais políticas de reorientação da formação profissional em saúde, bem como expandir 
essa pesquisa para explorar outras realidades e assegurar a consolidação da discussão, como compromisso político, na busca de profissionais mais aptos à colaboração e dispostos a enfrentarem, coletivamente, os problemas apresentados pela sociedade atual.

É essencial, portanto, que os ideais que fundamentam Sistema Único de Saúde (SUS) estimulem compromisso com a ampliação do debate em EIP na realidade brasileira, como um aliado importante para a superação de problemas históricos, que comprometem seriamente a qualidade da atenção à saúde no enfrentamento das complexas e dinâmicas necessidades de saúde da população.

\section{Referências}

1. Barr H. Interprofessional education: today, yesterday and tomorrow. A Review. 2005.

2. Hammick M, Freeth D, Koppel I, et al. A best evidence systematic review of interprofessional education: BEME Guide no. 9. Med. Teacher. 2007; 29(8):735-51.

3. Reeves S, Perrier L, Goldman J, et al. Interprofessional education: effects on professional practice and healthcare outcomes (update). Cochrane Database Syst Rev. 2013; 28(3):CD002213.

4. Centre for the Advancement of Interprofessional Education. The Centre for the Advancement of Interprofessional Education. CAIPE: United Kingdom; 2002.

5. Zwarenstein M, Goldman J, Reeves S. Interprofessional

\section{Colaboradores}

Costa MV (0000-0002-0573-0622)* contribuiu para a concepção, o planejamento, a análise e a interpretação dos dados; revisão crítica do conteúdo; e aprovação da versão final do manuscrito. Azevedo GD (0000-00027447-7712)* contribuiu para a concepção, a análise e a interpretação dos dados; revisão crítica do conteúdo; e aprovação da versão final do manuscrito. Vilar PJP (0000-00028765-8571)* contribuiu para a concepção, o planejamento, a análise e a interpretação dos dados; revisão crítica do conteúdo; e aprovação da versão final do manuscrito.

collaboration: effects of practice-based interventions on professional practice and healthcare outcomes. Cochrane Database Syst. Rev. 2009; 8(3):CD000072.

6. Bainbridge L, Wood VI. The power of prepositions: A taxonomy for interprofessional education. J Interp Care. 2012; 27(2):131-136.

7. Yin RK. Estudo de caso: planejamento e métodos. 2. ed. Porto Alegre: Bookman; 2009.

8. Turato ER. Métodos qualitativos e quantitativos na área da saúde: definições, diferenças e seus objetos de pesquisa. Rev. Saúde Pública. 2005; 39(3):507-514.

9. Gray DE. Pesquisa no mundo real. 2. ed. Porto Alegre: Penso; 2012.
*Orcid (Open Researcher and Contributor ID). 
10. Liamputtong P, Ezzy D. Qualitative Research Methods. New York, USA: Oxford University Press; 2005.

11. Dawson S, Manderson L, Tallo VL. A manual for the use of focus groups. INFDC: Boston; 1993.

12. Minayo MCS. O Desafio do Conhecimento: Pesquisa Qualitativa em Saúde. 11. ed. Rio de Janeiro: HUCITEC; 2008.

13. Barbour R. Grupos Focais. Porto Alegre: Artmed; 2009.

14. Bardin L. Análise de Conteúdo. Lisboa: Edições 70; 2009.

15. Cuff P, Schmitt M, Zierler B, et al. Interprofessional education for collaborative practice: views from a global forum workshop. J Interp Care. 2014; 28(1):2-4.

16. Xyrichis A, Ream E. Teamwork: a concept analysis. J Adv Nurs. 2008; 61(2):232-241.

17. Clements D, Dault M, Priest A. Effective Teamwork in Healthcare: Research and Reality. Healthcare Papers. 2007; 7(sp):26-34.

18. Mickan S, Hoffman SJ, Nasmith L. Collaborative practice in a global health context: Common themes from developed and developing countries. J Interp Care. 2010; 24(5):492-502.

19. Oandasan I, Reeves S. Key elements for interprofessional education. Part 1: The learner, the educator and the learning context. J Interp Care. 2005;19(sup1): 21-38.

20. Institute of Medicine. Interprofessional Education for Collaboration: Learning How to Improve Health from Interprofessional Models Across the Continuum of Education to Practice: Workshop Summary. Washington, DC: The National Academies Press; 2013.

21. Oandasan I, Reeves S. Key elements of interprofessional education. Part 2: Factors, processes and outcomes. Journal of Interprofessional Care. 2005; 19(sup1):39-48.

22. The New York Academy of Medicine. Interprofessional Care Coordination: Looking for The Future. Policy Research, \& Practice. 2013; 1(2):1-20.

23. Mellor R, Cottrell N, Moran M. "Just working in a team was a great experience..." - Student perspectives on the learning experiences of an interprofessional education program. J Interp Care. 2013; 27(4):292-297.

24. Weller J. Shedding new light on tribalism in health care. Med. Educ. 2012; 46(2):134-136.

25. Reeves S, Rice K, Conn LG, et al. Interprofessional interaction, negotiation and non-negotiation on general internal medicine wards. J Interp Care. 2009; 23(6):633-645.

26. Martimianakis MA, Maniate JM, Hodges BD. Sociological interpretations of professionalism. Med. Educ. 2009; 43(9):829-837.

27. Keddy B, Gillis MJ, Jacobs P, et al. The doctor-nurse relationship: an historical perspective. J Adv Nurs. 1986; 11(6):745-753.

28. Reeves S, Macmillan K, Van Soeren M. Leadership of interprofessional health and social care teams: a socio-historical analysis. Journal Nursing Manag. 2010; 18(3):258-264.

29. Baker MJ, Durham CF. Interprofessional Education: A Survey of Students' Collaborative Competency Outcomes. Journal Nursing Educ. 2013; 52(12):713-718.

30. Allen D. The nursing-medical boundary: a negotiated order? Socio. Health \& Illness. 1997; 19(4):498-520.

Recebido em 03/01/2019

Aprovado em 07/05/2019

Conflito de interesses: inexistente

Suporte financeiro: não houve 